\title{
Effect of Magnetic Field on Fluid Flow and Heat Transfer of a Nanofluid in a Vertical Channel with Heat Sources
}

\author{
${ }^{1}$ Madduru Sudhakara Reddy, ${ }^{2}$ Prof. A. Mallikarjuna Reddy \\ ${ }^{1}$ Research Scholar Department of Mathematics Sri Krishnadevaraya University, Anantapuramu, A.P., India \\ ${ }^{2}$ Professor of Mathematics Department of Mathematics Sri Krishnadevaraya University Anantapuramu, A.P., \\ India
}

*Corresponding Author: Madduru Sudhakara Reddy, Research Scholar Department of Mathematics

Sri Krishnadevaraya University, Anantapuramu, A.P., India

\begin{abstract}
We analyze the effect of magnetic field on Non Darcy convective heat and mass transfer flow of a nano fluid in vertical channel in presence of heat sources. The nonlinear governing equations have been solved by employing finite element technique. The velocity, Temperature and Concentration have been analyzed for different values of $M, \phi, \Delta$ and $\gamma$. The shear stress, rate of heat and mass transfer on the wall have been analyzed for different variations
\end{abstract}

Keywords: Vertical Channel, Heat Sources, Nano Fluid, Magnetic Field, Non-Darcy.

\section{INTRODUCTION}

The vertical channel is a frequently encountered configuration in thermal engineering equipment, for example, collectors of solar energy, cooling devices of electronic and micro-electronic equipments etc. The influence of electrically conducting the case of fully developed mixed convection between horizontal parallel plates with a linear axial temperature distribution was solved by Gill and Casal [13]. Ostrach [24] solved the problem of fully developed mixed convection between vertical plates with and without heat sources. Cebeci et al., [6] performed numerical calculations of developing laminar mixed convection between vertical parallel pates for both cases of buoyancy aiding and opposing conditions. Wirtz and McKinley [35] conducted an experimental study of a opposing mixed convection between vertical parallel plates with one plate heated and the other adiabatic. Several authors [Al-Nimir and Haddad [1], Greif et al [14] Gupta and Gupta [16], Datta and Jana [10], Barletta [2]. Barletta et al [3] Barletta et al [4], Maïga et al. [21], Maïga et al. [22], Polidori et al. [25], Hang and Pop [36], Maghrebi et al. [20], Grosan and Pop [15], Sacheti et al. [27], Fakour et al. [12], Nield and Kuznetsov [23], Sheikholeslami and Ganji [31], Das et al. [9], Rossi di Schio [26], Xu et al. [37]] have studied convective heat transfer different configurations. With the fuel crisis deepening all over the world, there is a great concern to utilize the enormous power beneath the earth's crust in the geothermal region. Liquid in the geothermal region is an electrically conducting liquid because of high temperature. Hence the study of interaction of the geomagnetic field with the fluid in the geothermal region is of great interest, thus leading to interest in the study of MHD convection flows through porous medium. Bharathi et al [11], Balasubramanyam et al [7 ] have discussed non darcy effect on convective heat and mass transfer flow in vertical channel under different conditions

Low thermal conductivity of conventional heat transfer fluids such as water, oil and ethylene glycol mixture is a primary limitation in enhancing the performance and the compactness of many engineering electronic devices. To overcome this drawback, there is a strong motivation to develop advanced heat transfer fluids with substantially higher conductivities to enhance thermal characteristics. Small particles (nanoparticles) stay suspended much longer than larger particles. If particles settle rapidly (micro particles), more particles need to be added to replace the settled particles, resulting in extra cost and degradation in the heat transfer enhancement. As such an innovative way in improving thermal conductivities of a fluid is to suspend metallic nanoparticles within it. The resulting mixture referred to as a nanofluid possesses a substantially larger thermal conductivity compared to that of traditional fluids. Nanofluids demonstrate anomalously high thermal 
conductivity, significant change in properties such as viscosity and specific heat in comparison to the base fluid, features which have attracted many researchers to perform in engineering applications. The popularity of nanofluids can be gauged from the researches done by scientists for its frequent applications and can be found in the literature [5,7,8,11,17-19,28-30,32,34,35]. Recently Sulochana et al [33] have discussed convective heat and mass transfer flow of a nano fluid in vertical channel in presence of heat sources and chemical reaction.

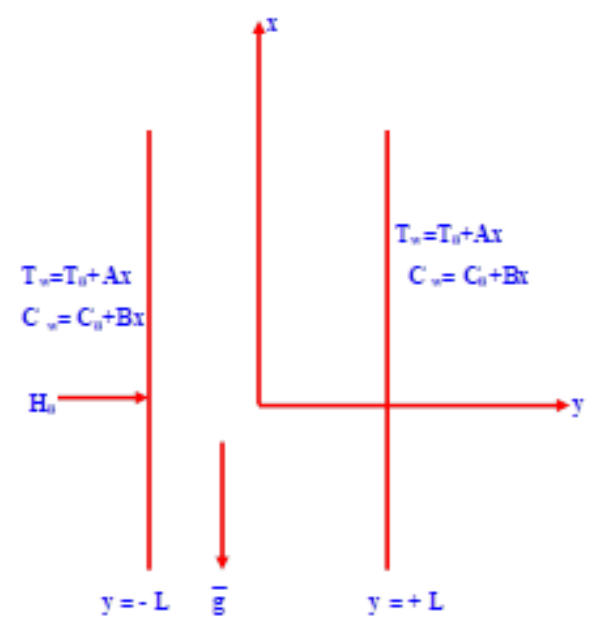

Fig1. Configuration of the problem

Keeping the above application in view we made an attempt to study thermo-diffusion and chemical reaction effects on non-Darcy convective heat and Mass transfer flow of a viscous electrically conducting nano fluid in a vertical channel with heat generating sources. The nonlinear governing equations have been solved by using Galerkin finite element analysis with quadratic approximation functions. The velocity, temperature, concentration, shear stress and rate of Heat and Mass transfer are evaluated numerically for different variations of parameter.

\section{Formulation of The Problem}

We consider a fully developed laminar convective heat and mass transfer flow of a nanofluid through a porous medium confined in a vertical channel bounded by flat walls. We choose a Cartesian coordinate system $\mathrm{O}(\mathrm{x}, \mathrm{y}, \mathrm{z})$ with $\mathrm{x}$ - axis in the vertical direction and $\mathrm{y}$-axis normal to the walls. The walls are taken at $y= \pm \mathrm{L}$. The walls are maintained at constant temperature and concentration. The temperature gradient in the flow field is sufficient to cause natural convection in the flow field. A constant axial pressure gradient is also imposed so that this resultant flow is a mixed convection flow.

The effective density of the nanofluid is given by

$\rho_{n f}=(1-\varphi) \rho_{f}+\varphi \rho_{s}$

Where $\phi$ is the solid volume fraction of nanoparticles

Thermal diffusivity of the nanofluid is

$\alpha_{n f}=\frac{k_{n f}}{\left(\rho C_{p}\right)_{n f}}$

Where the heat capacitance $C_{p}$ of the nanofluid is obtained as

$\left(\rho C_{p}\right)_{n f}=(1-\varphi)\left(\rho C_{p}\right)_{f}+\varphi\left(\rho C_{p}\right)_{s}$

And the thermal conductivity of the nanofluid $k_{n f}$ for spherical nanoparticles can be written as

$\frac{k_{n f}}{k_{f}}=\frac{\left(k_{s}+2 k_{f}\right)-2 \phi\left(k_{f}-k_{s}\right)}{\left(k_{s}+2 k_{f}\right)+\phi\left(k_{f}-k_{s}\right)}$

The thermal expansion coefficient of nanofluid can determine by 


$$
(\rho \beta)_{n f}=(1-\varphi)(\rho \beta)_{f}+\varphi(\rho \beta)_{s}
$$

Also the effective dynamic viscosity of the nanofluid given by

$$
\begin{aligned}
& \mu_{n f}=\frac{\mu_{f}}{(1-\varphi)^{2.5}} \\
& \sigma_{n f}=\sigma_{f}\left(1+\frac{3(\sigma-1) \phi}{\sigma+2-(\sigma-1) \phi}\right), \sigma=\frac{\sigma_{s}}{\sigma_{f}}
\end{aligned}
$$

Where the subscripts nf, $\mathrm{f}$ and s represent the thermo physical properties of the nanofluid, base fluid and the nanosolid particles respectively and $\phi$ is the solid volume fraction of the nanoparticles. The thermo physical properties of the nanofluid are given in Table 1.

\begin{tabular}{|l|l|l|l|l|}
\hline $\begin{array}{l}\text { Physical } \\
\text { Properties }\end{array}$ & Fluid phase & CuO (Copper)) & Al2O3 (Alumina) & TiO2 (Titanium dioxide) \\
\hline $\mathrm{Cp}(\mathrm{j} / \mathrm{kg} \mathrm{K})$ & 4179 & 385 & 765 & 686.2 \\
\hline$\rho\left(\mathrm{kg} \mathrm{m}^{3}\right)$ & 997.1 & 8933 & 3970 & 4250 \\
\hline $\mathrm{k}\left(\mathrm{W} / \mathrm{m} \mathrm{K}^{3}\right)$ & 0.613 & 400 & 40 & 8.9538 \\
\hline$\left.\beta \times 10^{-5} 1 / \mathrm{k}\right)$ & 21 & 1.67 & 0.63 & 0.85 \\
\hline
\end{tabular}

Since the flow is unidirectional, from the continuity of equation we find that

$$
\frac{\partial u}{\partial x}=0 \quad \text { where } \mathrm{u} \text { is the axial velocity implies } \mathrm{u}=\mathrm{u}(\mathrm{y})
$$

The momentum, energy and diffusion equations in the scalar form reduces to

$$
\begin{aligned}
& -\frac{\partial p}{\partial x}+\left(\frac{\mu_{n f}}{\delta}\right) \frac{\partial^{2} u}{\partial y^{2}}-\left(\frac{\sigma_{n f} \mu_{e}^{2} H_{o}^{2}}{\rho_{o}}\right) u-\frac{\rho_{n f} \delta F}{\sqrt{k}} u^{2}-\rho_{n f} g=0 \\
& \left(\rho_{0} C_{p}\right)_{n f} u \frac{\partial T}{\partial x}=k_{n f} \frac{\partial^{2} T}{\partial y^{2}}+Q\left(T_{o}-T\right)-\frac{\partial\left(q_{r}\right)}{\partial y} \\
& u \frac{\partial C}{\partial x}=D_{1} \frac{\partial^{2} C}{\partial y^{2}}-k_{1} C+k_{11} \frac{\partial^{2} T}{\partial y^{2}}
\end{aligned}
$$

The relevant boundary conditions are

$$
u=0, \quad \mathrm{~T}=\mathrm{T}_{\mathrm{w}}, \quad \mathrm{C}=\mathrm{C}_{\mathrm{w}} \text { at } \quad \mathrm{y}= \pm \mathrm{L}
$$

where $\mathrm{u}$ is the velocity, $\mathrm{T}, \mathrm{C}$ are the temperature and Concentration, $\mathrm{p}$ is the pressure,$\rho$ is the density of the fluid , $\mathrm{Cp}$ is the specific heat at constant pressure, $\mu$ is the coefficient of viscosity, $\mathrm{k}$ is the permeability of the porous medium, $\delta$ is the porosity of the medium, $\beta$ is the coefficient of thermal expansion, $\mathrm{k}_{\mathrm{f}}$ is the coefficient of thermal conductivity, $\mathrm{F}$ is a function that depends on the Reynolds number and the microstructure of porous medium, $\beta^{\bullet}$ is the volumetric coefficient of expansion with mass fraction concentration, $\mathrm{k}_{1}$ is the chemical reaction coefficient and $\mathrm{D}_{1}$ is the chemical molecular diffusivity, $\mathrm{q}_{\mathrm{R}}$ is the radiative heat flux, $\mathrm{k}_{11}$ is the cross diffusivity, $\mathrm{Q}$ is the strength of the heat generating source,. Here, the thermophysical properties of the solid and fluid have been assumed to be constant except for the density variation in the body force term (Boussinesq approximation) and the solid particles and the fluid are considered to be in the thermal equilibrium) .

We assume that the temperature and concentration of the both walls is $T_{w}=T_{0}+A x, C_{w}=C_{0}+B x$ where $\mathrm{A}$ and $\mathrm{B}$ are the vertical temperature and concentration gradients which are positive for buoyancy -aided flow and negative for buoyancy -opposed flow, respectively, $T_{0}$ and $C_{0}$ are the upstream reference wall temperature and concentration respectively. For the fully developed laminar flow in the presences of radial magnetic field, the velocity depend 
only on the radial coordinate and all the other physical variables except temperature, concentration and pressure are functions of $\mathrm{y}$ and $\mathrm{x}, \mathrm{x}$ being the vertical co-ordinate

The temperature and concentration inside the fluid can be written as

$T=T^{\bullet}(y)+A x \quad, \quad C=C^{\bullet}(y)+B x$

We define the following non-dimensional variables as

$$
\begin{aligned}
& u^{\prime}=\frac{u}{(v / L)},\left(x^{\prime}, y^{\prime}\right)=(x, y) / L, \quad p^{\prime}=\frac{p \delta}{\left(\rho v^{2} / L^{2}\right)} \\
& \theta(\mathrm{y})=\frac{\mathrm{T}^{\bullet}-\mathrm{T}_{0}}{\mathrm{ALP}_{1}}, \quad, \quad \mathrm{C}=, \frac{\mathrm{C}^{\bullet}-\mathrm{C}_{0}}{\mathrm{BLP}_{1}} \\
& A_{1}=\frac{1}{(1-\varphi)^{2.5}}, \quad A_{2}=(1-\varphi)+\varphi\left(\frac{\rho_{s}}{\rho_{f}}\right), \\
& A_{3}=1-\varphi+\varphi \frac{\left(\rho C_{p}\right)_{s}}{\left(\rho C_{p}\right)_{f}}, A_{4}=1-\varphi+\varphi\left(\frac{(\rho \beta)_{s}}{(\rho \beta)_{f}}, \quad A_{5}=\frac{k_{n f}}{k_{f}}, A_{6}=\left(1+\frac{3(\sigma-1) \phi}{\sigma+2-(\sigma-1) \phi}\right)\right.
\end{aligned}
$$

Introducing these non-dimensional variables the governing equations in the dimensionless form reduce to (on dropping the dashes)

$A_{1} \frac{d^{2} u}{d y^{2}}=1+\delta\left(A_{6} M^{2}+A_{1} D^{-1}\right) u-\delta G A_{4}(\theta)-\delta^{2} A_{2} \Delta u^{2}$

$A_{5} \frac{d^{2} \theta}{d y^{2}}-\alpha \theta=\left(A_{3} P\right) u$

$\frac{d^{2} C}{d y^{2}}-(\gamma S c) C=(S c) u$

where

$\Delta=F D^{-1 / 2}$ (Inertia or Fochhemeir parameter), $G=\frac{\beta_{f} g A L^{3}}{v_{f}^{2}}$ (Grashof Number)

$M^{2}=\frac{\sigma_{f} \mu_{e}^{2} H_{o}^{2} L^{2}}{v_{f}^{2}}$ (Hartmann Number), $S c=\frac{v}{D_{B}}$ (Schmidt number $)$

$P=\frac{\mu_{f} C_{p f}}{k_{f}}$ (Prandtl Number), $\alpha=\frac{Q L^{2}}{k_{f}}$ (Heat source parameter)

$\gamma=\frac{k_{1} L^{2}}{v_{f}} \quad$ (Chemical reaction parameter)

The corresponding boundary conditions are

$u=0, \theta=0, C=0$ on $y= \pm 1$

\section{Finite Element AnAlysis}

The finite element analysis with quadratic polynomial approximation functions is carried out to solved the equations (12-14). The behavior of the velocity, temperature and concentration profiles has been discussed computationally for different variations in governing parameters. The Gelarkin method has 
been adopted in the variational formulation in each element to obtain the global coupled matrices for the velocity, temperature and concentration in course of the finite element analysis.

Choose an arbitrary element $e_{k}$ and let $u^{k}, \theta^{k}$ and $C^{k}$ be the values of $u, \theta$ and $C$ in the element $e_{k}$. We define the error residuals as

$$
\begin{aligned}
& E_{u}^{i}=\frac{d}{d \eta}\left(A_{1} \frac{d u^{i}}{d \eta}\right)-\left(A_{6} M^{2}+A_{1} D^{-1}\right) u^{i}+\delta^{2} A_{2} \mathrm{~A}\left(u^{i}\right)^{2}-\delta A_{4} G\left(\theta^{i}\right) \\
& E_{c}^{i}=\frac{d}{d y}\left(\frac{d C^{i}}{d y}\right)-(\gamma S c) C^{i}-S c u^{i} \\
& E_{\theta}^{i}=\frac{d}{d y}\left(A_{5} \frac{d \theta^{i}}{d y}\right)-\alpha \theta^{i}=\left(A_{3} P u^{i}\right)
\end{aligned}
$$

where $u^{k}, \theta^{k} \& C^{k}$ are values of $u, \theta \& C$ in the arbitrary element $e_{k}$. These are expressed as linear combinations in terms of respective local nodal values.

$u^{k}=u_{1}^{k} \psi_{1}^{k}+u_{2}^{k} \psi_{1}^{k}+u_{3}^{k} \psi_{3}^{k}, \theta^{k}=\theta_{1}^{k} \psi_{1}^{k}+\theta_{2}^{k} \psi_{2}^{k}+\theta_{3}^{k} \psi_{3}^{k}, C^{k}=C_{1}^{k} \psi_{1}^{k}+C_{2}^{k} \psi_{2}^{k}+C_{3}^{k} \psi_{3}^{k}$

where $\psi_{1}^{k}, \psi_{2}^{k}$--------- etc are Lagrange's quadratic polynomials.

Galerkin's method is used to convert the partial differential Eqs. (16) - (18) into matrix form of equations which results into $3 \times 3$ local stiffness matrices. All these local matrices are assembled in a global matrix by substituting the global nodal values of order I and using inter element continuity and equilibrium conditions.

The shear stress $(\tau)$, Nusselt number (rate of heat transfer), Sherwood number (rate of mass transfer) are evaluated by using the following formulas

$$
\tau_{y= \pm L}=\mu\left(\frac{d u}{d y}\right)_{y= \pm L} \quad N u_{y= \pm i}=\left(\frac{d \theta}{d y}\right)_{y= \pm 1} S h_{y= \pm 1}=\left(\frac{d C}{d y}\right)_{y= \pm 1}
$$

\begin{tabular}{|c|c|c|c|c|c|c|c|c|c|}
\hline \multirow{3}{*}{\multicolumn{2}{|c|}{$\begin{array}{l}\text { Para } \\
\text { meter }\end{array}$}} & \multirow{2}{*}{\multicolumn{2}{|c|}{$\begin{array}{l}\text { Sulochana et al [33] } \\
\text { Cuo-water }\end{array}$}} & \multirow{2}{*}{\multicolumn{2}{|c|}{$\begin{array}{l}\text { Present Results } \\
\text { Cuo-water }\end{array}$}} & \multirow{2}{*}{\multicolumn{2}{|c|}{$\begin{array}{l}\text { Sulochana et al [33] } \\
\mathrm{Al}_{2} \mathrm{O}_{3}\end{array}$}} & \multirow{2}{*}{\multicolumn{2}{|c|}{$\begin{array}{l}\text { Present Results } \\
\mathrm{Al}_{2} \mathrm{O}_{3}\end{array}$}} \\
\hline & & & & & & & & & \\
\hline & & $\tau(-1)$ & $\tau(+1)$ & $\tau(-1)$ & $\tau(+1)$ & $\tau(-1)$ & $\tau(+1)$ & $\tau(-1)$ & $\tau(+1)$ \\
\hline \multirow[t]{4}{*}{$\phi$} & 0.05 & 0.0162093 & -0.0142988 & 0.0162113 & -0.0142928 & 0.0119033 & -0.0104835 & 0.0119023 & -0.0104815 \\
\hline & 0.1 & 0.0310306 & -0.0272453 & 0.0310296 & -0.0272473 & 0.0074749 & -0.0082180 & 0.0074699 & -0.0082160 \\
\hline & 0.3 & 0.0466166 & -0.0403833 & & -0.0403843 & 0.0064953 & -0.0079705 & 0.0064923 & -0.0079665 \\
\hline & 0.5 & 0.0512127 & -0.0502972 & 0.051 & -0.0502982 & 0.0054132 & -0.0068427 & 0.0054102 & -0.0068407 \\
\hline \multirow[t]{4}{*}{$\Delta$} & 2 & 0.0162093 & -0.0142988 & 0.0162083 & -0.0142998 & 0.0119033 & -0.0104835 & 0.0119023 & -0.0104805 \\
\hline & 4 & 0.0171074 & -0.0162075 & 0.0171084 & -0.0162065 & 0.0150951 & -0.0125026 & 0.0150961 & -0.0125006 \\
\hline & 6 & 0.0391748 & -0.0344822 & 0.0391728 & -0.0344792 & 0.0251264 & -0.0210956 & 0.0251224 & -0.0210906 \\
\hline & 10 & 0.0854013 & -0.0753128 & 0.0854003 & -0.0753108 & 0.0550952 & -0.0452329 & 0.0550922 & -0.0452299 \\
\hline
\end{tabular}

\begin{tabular}{|c|c|c|c|c|c|c|c|c|c|}
\hline \multirow{3}{*}{\multicolumn{2}{|c|}{ Parameter }} & \multirow{2}{*}{\multicolumn{2}{|c|}{$\frac{\text { Sulochana et al [33] }}{\text { Cuo-water }}$}} & \multirow{2}{*}{\multicolumn{2}{|c|}{$\begin{array}{c}\text { Present Results } \\
\text { Cuo-water }\end{array}$}} & \multirow{2}{*}{\multicolumn{2}{|c|}{$\begin{array}{c}\text { Sulochana et al [33] } \\
\mathrm{Al}_{2} \mathrm{O}_{3}\end{array}$}} & \multirow{2}{*}{\multicolumn{2}{|c|}{$\begin{array}{c}\text { Present Results } \\
\mathrm{Al}_{2} \mathrm{O}_{3}\end{array}$}} \\
\hline & & & & & & & & & \\
\hline & & $\mathrm{Nu}(-1)$ & $\mathrm{Nu}(+1)$ & $\mathrm{Nu}(-1)$ & $\mathrm{Nu}(+1)$ & $\mathrm{Nu}(-1)$ & $\mathrm{Nu}(+1)$ & Nu (-1) & $\mathrm{Nu}(+1)$ \\
\hline \multirow[t]{4}{*}{$\phi$} & 0.05 & -0.067302 & -0.104039 & -0.067292 & -0.104029 & -0.015596 & -0.241131 & -0.015546 & -0.241101 \\
\hline & 0.1 & -0.072895 & -0.199393 & -0.072925 & -0.199363 & -0.006479 & -0.100062 & -0.006429 & -0.100042 \\
\hline & 0.3 & -0.087433 & -0.300521 & -0.087463 & -0.300491 & -0.005799 & -0.090438 & -0.005729 & -0.090398 \\
\hline & 0.5 & -0.091245 & -0.354291 & -0.091265 & -0.354261 & -0.005019 & -0.086120 & -0.005009 & -0.086100 \\
\hline \multirow[t]{4}{*}{$\Delta$} & 2 & -0.067302 & -0.104039 & -0.067292 & -0.104029 & -0.015596 & -0.241131 & -0.015526 & -0.241111 \\
\hline & 4 & -0.068168 & -0.110345 & -0.068098 & -0.110325 & -0.016751 & -0.256795 & -0.016721 & -0.256725 \\
\hline & 6 & -0.068894 & -0.126113 & -0.068914 & -0.126103 & -0.017503 & -0.264691 & -0.017463 & -0.264661 \\
\hline & 10 & -0.070874 & -0.168983 & -0.070894 & -0.168963 & -0.019441 & -0.302593 & -0.019421 & -0.302553 \\
\hline
\end{tabular}

Comparison: In the absence of magnetic field $(\mathrm{M}=0)$ and thermal radiation $(\mathrm{Rd}=0)$ the results are in good agreement with Sulochana et al [33] 


\section{DiSCUSSION OF THE NUMERICAL RESUlTS}

A mathematical assessment for the numerical solution of this problem is performed, and the outcomes are illustrated graphically in Figures 2- 5. They explain the fascinating features of important parameters on the nanofluid velocity, temperature, concentration distributions, skin friction, Nusselt number and Sherwood number for two different types of water based nanofluids. We take the values of the nanofluid volume fraction $\phi$ in the range of $0 \leq \phi \leq 0.08$. We considered for the convective flow in a lid driven cavity, the value of the nanofluid volume fraction in the range $0 \leq \phi \leq 0.08$. If the concentration exceeds the maximum level of 0.08 , sedimentation could take place. We have chosen here $\operatorname{Pr}=6.2$ while $\mathrm{M}, \Delta, \gamma, \phi$ are varied over a range, which are listed in the Figure legends.

Fig.2a represents the effect of magnetic parameter $\mathrm{M}$ on the nanofluid velocity profile. It is observed from the figure that the velocity distribution decreases with increasing magnetic parameter $\mathrm{M}$. It is found that an increase in the magnetic parameter $M$ reduces the velocity throughout the flow region. This reduction can be attributed to the fact that the magnetic field provides a resistive type of force known as the Lorentz force. This force tends to lessen the motion of the fluid as a consequence the velocity reduces. We also find that the nano-fluid velocity in the case of $\mathrm{CuO}$ - water nanofluid is relatively greater than that of $\mathrm{Al}_{2} \mathrm{O}_{3}$-water nanofluid. This phenomenon has good agreement with the physical realities. Fig. $2 \mathrm{~b}$ represents the effect of magnetic parameter $\mathrm{M}$ on the nanofluid temperature profile. It is observed from the figure that the temperature distribution increases with increasing values of $\mathrm{M}$ as a result of increase in the thickness of the thermal boundary layer owing to the Lorentz force developed by the magnetic field.. We also find that the nano-fluid temperature in the case of $\mathrm{CuO}$ - water nanofluid is relatively greater than that of $\mathrm{Al}_{2} \mathrm{O}_{3}$-water nanofluid. This phenomenon has good agreement with the physical realities Fig.2c represents the effect of $M$ on the nanofluid concentration profile. It is observed from the figure that the concentration distribution increases with increasing values of $\mathrm{M}$ as a result of enhancement of the thickness of the solutal boundary layer. We also find that the nano-fluid concentration in the case of $\mathrm{CuO}$-water nanofluid is relatively greater than that of $\mathrm{Al}_{2} \mathrm{O}_{3}$-water nanofluid. This phenomenon has good agreement with the physical realities.

Figs.3a\&3b represent the concentration (C) with chemical reaction parameter $\gamma$.It can be seen from the concentration profiles that the concentration reduces in both degenerating and generating chemical reaction cases.Also the values of the concentration in $\mathrm{Al}_{2} \mathrm{O}_{3}$-water nanofluid are relatively smaller than those of Cuo-water nanofluid.

Figs.4a depicts the behaviour of velocity with Forchhemeir parameter $\Delta$.It can be seen from the profiles that reduces with increasing Forchhemeir number $\Delta$ in both types of nanofluids. The values of velocity in Cuo-water are relatively greater than those of $\mathrm{Al}_{2} \mathrm{O}_{3}$-water nanofluid. From fig. $4 \mathrm{~b}$ we find that the temperature reduces wit increasing $\Delta$ in both types of Nanofluids. It can be attributed to the fact that increase in Forchhemeir parameter $\Delta$ reduces the thickness of the thermal boundary layer. From fig. $4 \mathrm{c}$ we notice that the solutal concentration reduces with increase in $\Delta$.This may be attributed to the fact that the thickness of the solutal boundary layer reduces with increasing $\Delta$. Also the values of concentration in Al203-water nanofluid are relatively lesser than those of Cuo-water nanofluid.

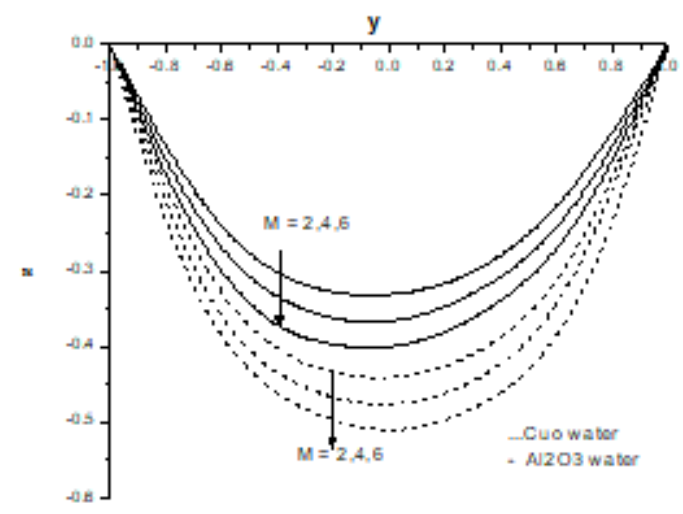

Fig2a. Variation of $\mu$ with $M \gamma=0.5, \Delta=0.2, \phi=0.05$

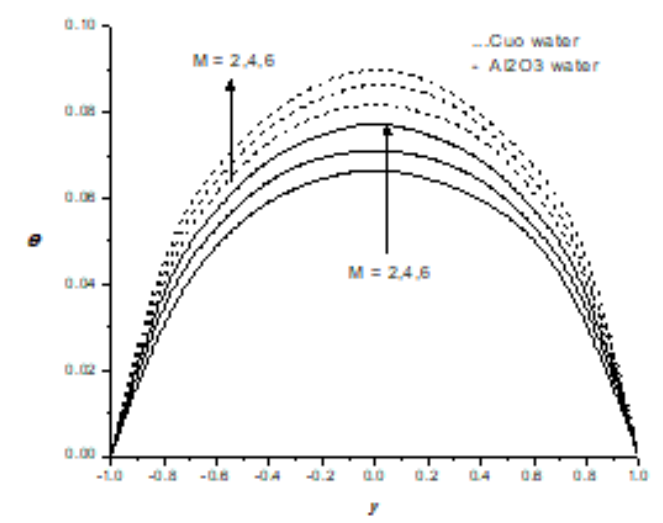

Fig2b. Variation of $\theta$ with $M \gamma=0.5, \Delta=0.2, \phi=0.05$ 


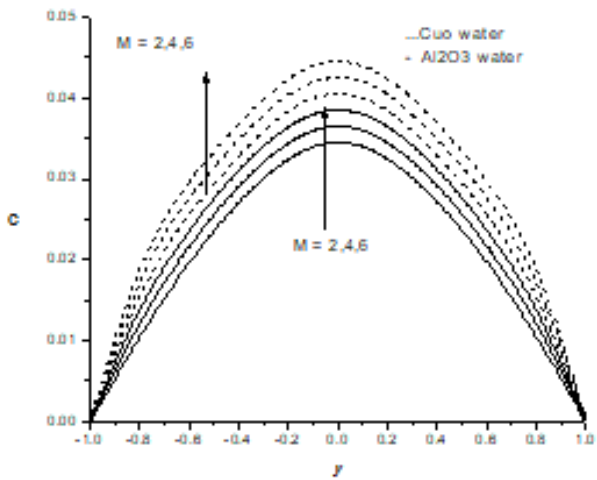

Fig2c. Variation of $C$ with $M \gamma=0.5, \Delta=0.2, \phi=0.05$

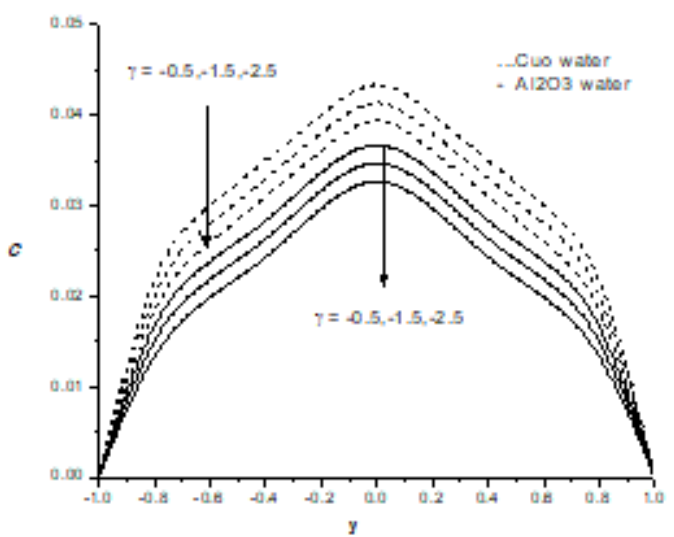

Fig3b. Variation of $C$ with $\gamma<0 M=2, \Delta=0.2, \phi=0.05$

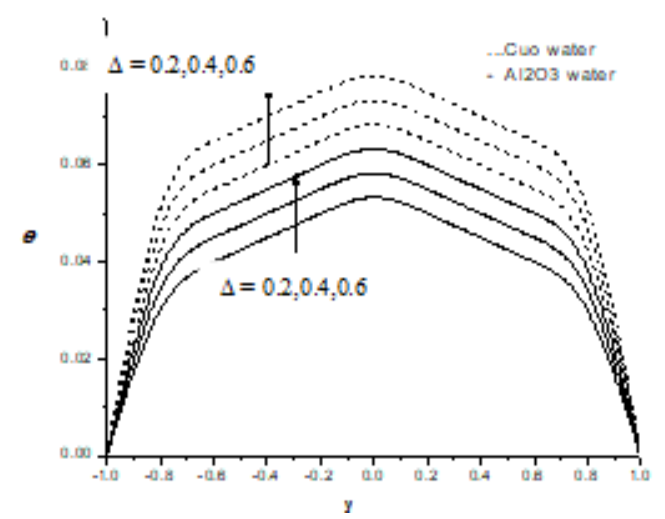

Fig4b. Variation of $\theta$ with $\Delta M=2, \gamma=0.5, \phi=0.05$

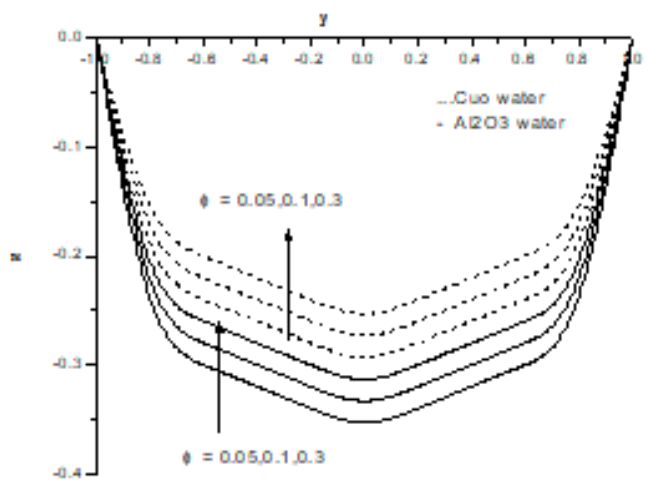

Fig5a. Variation of $u$ with $\phi M=2, \gamma=0.5, \Delta=0.2$

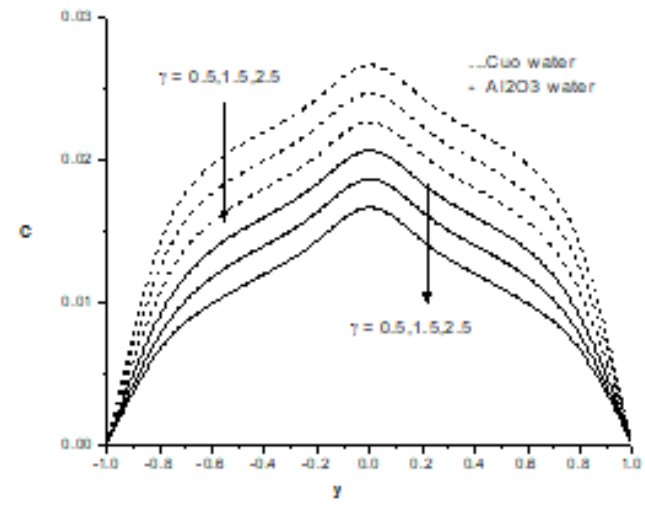

Fig3a. Variation of $C$ with $\gamma>0 M=2, \Delta=0.2, \phi=0.05$

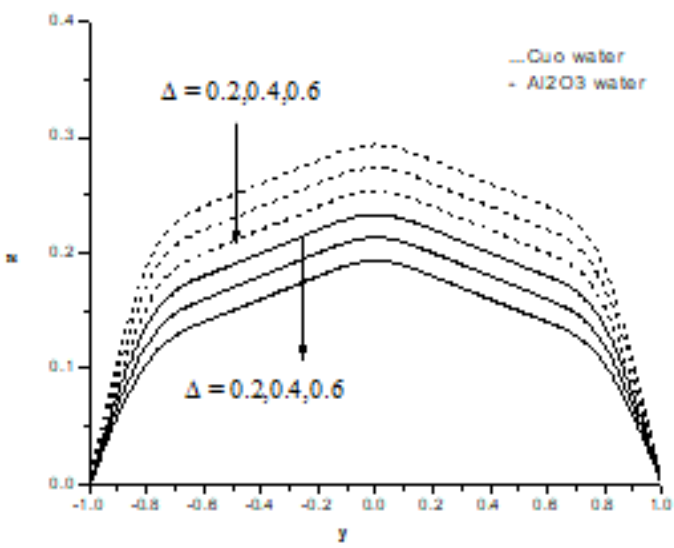

Fig4a. Variation of $u$ with $\Delta M=2, \gamma=0.5, \phi=0.05$

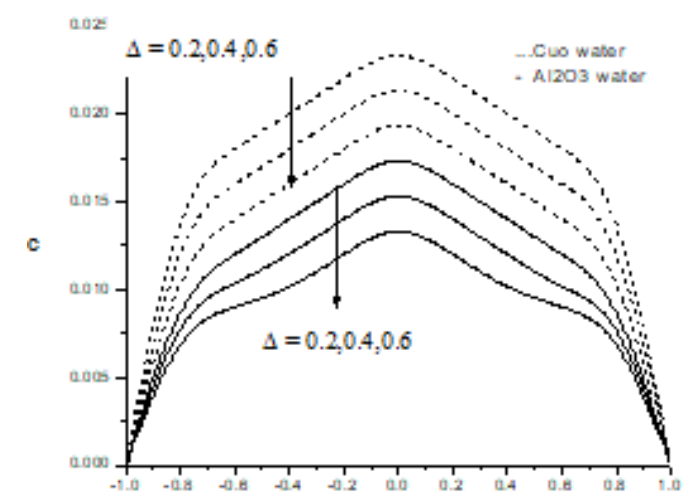

Fig4c. Variation of $C$ with $\Delta M=2, \gamma=0.5, \phi=0.05$

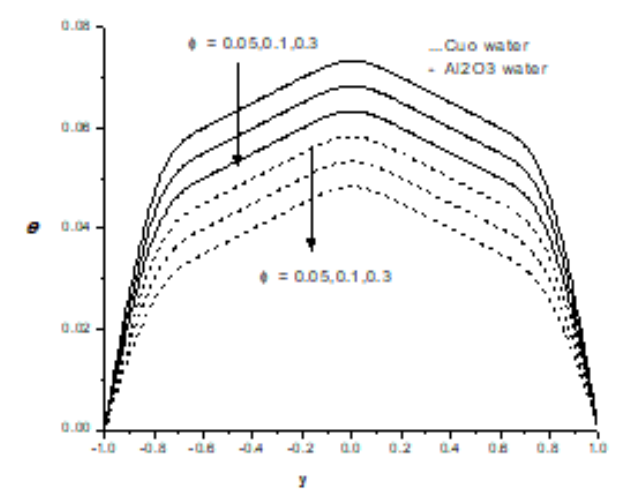

Fig5b. Variation of $\theta$ with $\phi M=2, \gamma=0.5, \Delta=0.2$ 


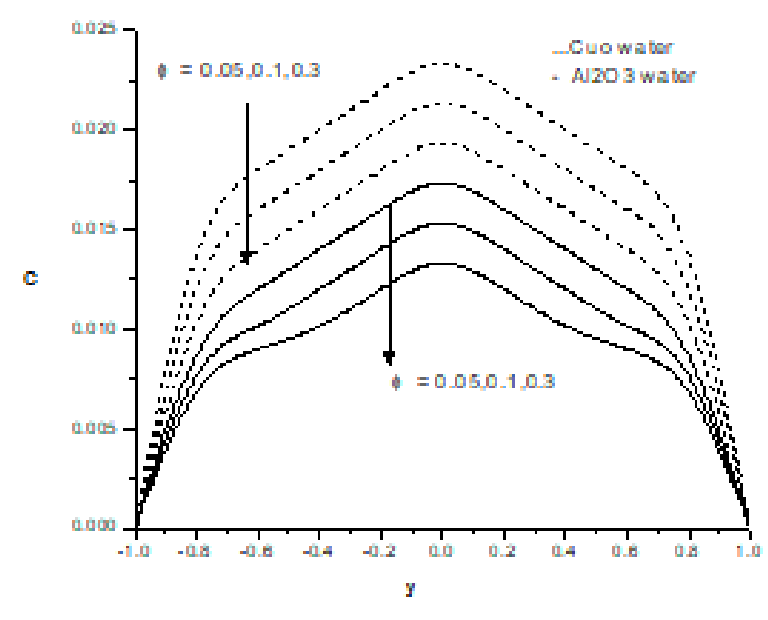

Fig5c. Variation of $C$ with $\phi M=2, \gamma=0.5, \Delta=0.2$

Figs.5a display the effect of nanoparticle volume fraction $\phi$ on the nanofluid velocity and. It is found that as the nanoparticle volume fraction increases the nanofluid velocity experiences an enhancement in the boundary layer in the case of Cuo0water nanofluid and reduces in $\mathrm{Al}_{2} \mathrm{O}_{3}$-water nanofluid. These Figures illustrate this agreement with the physical behaviour. When the volume of the nanoparticle increases the thermal conductivity and the thermal boundary layer thickness increases. We also notice that the nanofluid velocity in the case of $\mathrm{Al}_{2} \mathrm{O}_{3}$ - water nanofluid is relatively lesser than that of $\mathrm{CuO}-$ water nanofluid. Fig.5b shows that the variation of temperature with $\phi$. It can be seen from the profiles that an increase in the nanoparticle volume fraction reduces the temperature in the boundary layer. This is due to the fact that the thickness of the thermal boundary layer decreases with increase in $\phi$. Also we find that the temperature in $\mathrm{Al}_{2} \mathrm{O}_{3}$-water is relatively lesser than that of $\mathrm{CuO}$-water fluid. Fig.5c shows the variation of concentration with nanoparticle volume fraction $\phi$. We notice a reduction in the concentration with increasing $\phi$. This may be attributed to the fact that an enhancement in $\phi$ results in decreasing the thickness of the solutal boundary layer. The concentration in $\mathrm{CuO}$-water nanofluid is higher than those values of $\mathrm{C}$ in $\mathrm{Al}_{2} \mathrm{O}_{3}$-water nano fluid.

The table displays the behavior of local skin friction $(\tau)$ at the walls $y= \pm 1$. With respect to the Forchheimir parameter A shows that the skin friction reduces with increase in $\Delta$. Thus the inclusion of inertial and boundary effects reduces the skin friction at both the walls in both types of nanofluids. An increase in the nano particle volume fraction $\phi$ reduces $\tau$ at the walls in $\mathrm{CuO}$-water nanofluid and $\mathrm{Al}_{2} \mathrm{O}_{3}$-water nanofluid. From the tabular values of the skin friction w.r.to different parametric variations we find that the magnitude of skin friction in $\mathrm{CuO}$-water nanofluid is relatively greater than those values in $\mathrm{Al}_{2} \mathrm{O}_{3}$-water nanofluid.In all the variations, we find that the values of skin friction in Cuo-water nanofluid are relatively larger than those in $\mathrm{Al}_{2} \mathrm{O}_{3}$-water nanofluid .

The local Nusselt number $(\mathrm{Nu})$ at the walls $\mathrm{y}= \pm 1$ is shown in table for different parametric variations. Magnetic parameter $M$ shows that higher the Lorentz force larger the rate of heat transfer at $y= \pm 1$. An increase in the Nanoparticle volume fraction $\phi$ reduces $\mathrm{Nu}$ at $\mathrm{y}= \pm 1$ in $\mathrm{CuO}$-water nanofluid and $\mathrm{Al}_{2} \mathrm{O}_{3}$-water nanofluids. The rate of heat transfer in the $\mathrm{CuO}$-water nanofluid is relatively lesser than those values in $\mathrm{Al}_{2} \mathrm{O}_{3}$-water nanofluid. With reference to the Forchheimir parameter $\Delta$,we find that $|\mathrm{Nu}|$ reduces the rate of heat transfer at both the walls in both types of nanofluids. Thus the inclusion of inerial and boundary effects results in a reduction in $|\mathrm{Nu}|$ at both walls. Also we notice that the rate of heat transfer in Cuo-water nanofluid is relatively smaller than those in $\mathrm{Al}_{2} \mathrm{O}_{3}$-water nanofluid

The table represents the variation of mass transfer Sh at $y= \pm 1$ with different values of $M, \gamma, \Delta$ and $\phi$. The variation of Sh with Chemical reaction parameter $\gamma$ shows that $|\mathrm{Sh}|$ at $\mathrm{y}=+1$ reduces in $\mathrm{CuO}$-water nanofluid and enhances it in $\mathrm{Al}_{2} \mathrm{O}_{3}$-water nanofluid in degenerating chemical reaction case, while in generating chemical reaction case $|\mathrm{Sh}|$ enhances in $\mathrm{CuO}$-water nanofluid and reduces in $\mathrm{Al}_{2} \mathrm{O}_{3}$-water nanofluid. In both the degenerating and generating chemical reaction case we find that the vales of $|\mathrm{Sh}|$ at $\mathrm{y}=+1$ in $\mathrm{CuO}$-water nanofluid are relatively greater than those values in $\mathrm{Al}_{2} \mathrm{O}_{3}$-water nanofluid. At $y=-1,|\mathrm{Sh}|$ enhances in degenerating case and reduces in generating case. The values of Sh for $\gamma>0$ 
in $\mathrm{CuO}$-water nanofluid are relatively lesser than those values in $\mathrm{Al}_{2} \mathrm{O}_{3}$-water nanofluid, While a reversed effect is noticed in the behaviour of $|\mathrm{Sh}|$. The variation of Sh with Nano particle volume fraction $\phi$ shows that the rate of mass transfer enhances at $y=+1$ and recues at $y=-1$ with increase in $\phi$ in $\mathrm{CuO}$-water nanofluid. In $\mathrm{Al}_{2} \mathrm{O}_{3}$-water nanofluid $|\mathrm{Sh}|$ reduces at $\mathrm{y}=+1$ and enhances at $\mathrm{y}=-1$. Also we notice that the values of $|\mathrm{Sh}|$ at $\mathrm{y}=+1$ in $\mathrm{CuO}$-water nanofluid are relatively greater than those of $\mathrm{Al}_{2} \mathrm{O}_{3}$-water nanofluid,while at $\mathrm{y}=-1|\mathrm{Sh}|$ in $\mathrm{CuO}$-water nanofluid are relatively lesser than those of $\mathrm{Al}_{2} \mathrm{O}_{3}$-water nanofluid.

Table2. Skin friction $(\tau)$ at the walls $y= \pm 1$

\begin{tabular}{|c|c|c|c|c|c|}
\hline \multirow{2}{*}{\multicolumn{2}{|c|}{ Parameter }} & \multicolumn{2}{|c|}{ Cuo-water } & \multicolumn{2}{|c|}{$\mathrm{Al}_{2} \mathrm{O}_{3}$-water } \\
\hline & & \multirow{2}{*}{$\tau(\mathbf{- 1})$} & \multirow{2}{*}{$\begin{array}{c}\tau(\mathbf{1}) \\
1.00089\end{array}$} & \multirow{2}{*}{$\begin{array}{c}\tau(\mathbf{- 1}) \\
-0.99747\end{array}$} & \multirow{2}{*}{$\begin{array}{c}\tau(\mathbf{1}) \\
0.997471\end{array}$} \\
\hline $\mathbf{M}$ & 0.5 & & & & \\
\hline & 1.2 & -1.02195 & 1.02195 & -1.02195 & 1.02195 \\
\hline & 1.5 & -1.06742 & 1.06742 & -1.06742 & 1.06742 \\
\hline & 3.0 & -1.14551 & 1.14551 & -1.14551 & 1.14551 \\
\hline \multirow[t]{4}{*}{$\Delta$} & 0.05 & -1.00089 & 1.00089 & -0.99747 & 0.99747 \\
\hline & 0.5 & -1.00083 & 1.00083 & -1.00083 & 1.00083 \\
\hline & 1.0 & -1.00076 & 1.00076 & -1.00076 & 1.00076 \\
\hline & 1.5 & -1.00063 & 1.00063 & -1.00063 & 1.00063 \\
\hline \multirow[t]{4}{*}{$\phi$} & 0.05 & -1.00089 & 1.00089 & -0.99747 & 0.99747 \\
\hline & 0.1 & -1.00038 & 1.00083 & -1.00083 & 1.00083 \\
\hline & 0.3 & -1.00008 & 1.00076 & -1.00076 & 1.00076 \\
\hline & 0.5 & -0.99992 & 1.00063 & -1.00063 & 1.00063 \\
\hline
\end{tabular}

Table3. Nusselt Number $(N u)$ at the walls $y= \pm 1$

\begin{tabular}{|c|c|c|c|c|c|}
\hline \multirow{2}{*}{\multicolumn{2}{|c|}{ Parameter }} & \multicolumn{2}{|c|}{ Cuo-water } & \multicolumn{2}{|c|}{ Al203 -water } \\
\hline & & Nu (-1) & $\mathrm{Nu}(1)$ & \multirow{2}{*}{$\begin{array}{c}\text { Nu (-1) } \\
-0.0182479\end{array}$} & \multirow{2}{*}{$\begin{array}{c}\text { Nu (1) } \\
0.0182479\end{array}$} \\
\hline $\mathrm{M}$ & 0.5 & -0.0184921 & 0.0184924 & & \\
\hline & 1.0 & -0.0185729 & 0.0185729 & -0.0185517 & 0.0185517 \\
\hline & 1.5 & -0.0187468 & 0.0187468 & -0.0187245 & 0.0187245 \\
\hline & 3.0 & -0.0190217 & 0.0190217 & -0.0190217 & 0.0190217 \\
\hline \multirow[t]{4}{*}{$\Delta$} & 0.05 & -0.0184718 & 0.0184718 & -0.0182479 & 0.0182479 \\
\hline & 0.5 & -0.0184715 & 0.0184715 & -0.0184715 & 0.0184715 \\
\hline & 1.0 & -0.0184712 & 0.0184712 & -0.0184712 & 0.0184712 \\
\hline & 1.5 & -0.0184707 & 0.0184707 & -0.0184707 & 0.0184707 \\
\hline \multirow[t]{4}{*}{$\phi$} & 0.05 & -0.0184718 & 0.0184718 & -0.0182479 & 0.0182479 \\
\hline & 0.1 & -0.0184698 & 0.0184698 & -0.0184698 & 0.0184698 \\
\hline & 0.3 & -0.0184687 & 0.0184687 & -0.0184687 & 0.0184687 \\
\hline & 0.5 & -0.0184681 & 0.0184681 & -0.0184681 & 0.0184681 \\
\hline
\end{tabular}

Table4. Sherwood number(Sh) at the walls $y= \pm 1$

\begin{tabular}{|c|c|c|c|c|c|}
\hline \multicolumn{2}{|c|}{ Parameter } & \multicolumn{2}{c|}{ Cuo-water } & \multicolumn{2}{c|}{ Al2O3-water } \\
\cline { 2 - 6 } M & & Sh (1) & Sh (2) & Sh (1) & Sh (2) \\
& 0.5 & -0.067479 & 0.067479 & -0.067201 & 0.0672018 \\
& 1.0 & -0.069180 & 0.0691809 & -0.069180 & 0.0691809 \\
& 1.5 & -0.072859 & 0.0728595 & -0.072859 & 0.0728595 \\
& 3.0 & -0.075293 & 0.0752939 & -0.079185 & 0.0791855 \\
\hline$\gamma$ & 0.5 & -0.070318 & 0.0703186 & -0.067201 & 0.0672018 \\
& 1.5 & -0.064862 & 0.0648624 & -0.062443 & 0.0624435 \\
& -0.5 & -0.076791 & 0.0767917 & -0.080502 & 0.0805029 \\
& -1.5 & -0.079998 & 0.0799989 & -0.089132 & 0.089132 \\
\hline \multirow{4}{*}{$\gamma$} & 0.05 & -0.067479 & 0.067479 & -0.067201 & 0.0672018 \\
& 0.5 & -0.067473 & 0.0674732 & -0.067473 & 0.0674732 \\
& 1.0 & -0.067467 & 0.0674675 & -0.067467 & 0.0674675 \\
& 1.5 & -0.067455 & 0.0674559 & -0.067455 & 0.0674559 \\
\hline \multirow{2}{*}{$\phi$} & 0.05 & -0.067479 & 0.067479 & -0.067201 & 0.0672018 \\
& 0.1 & -0.067437 & 0.0674379 & -0.067437 & 0.0674379 \\
& 0.3 & -0.067413 & 0.0674135 & -0.067413 & 0.0674135 \\
& 0.5 & -0.0674007 & 0.0674007 & -0.067407 & 0.0674007 \\
\hline
\end{tabular}




\section{CONClusions}

The non-linear coupled equations governing the flow, heat and mass transfer have been solved by employing Galerkine finite element technique with quadratic polynomials. The important conclusions of this analysis are

- The velocity decreases while the temperature and concentration enhances with increasing $M$. The skin friction and Nusselt number increases with increasing $M$ at both the walls.

- An increase in the strength of the heat generating source reduce the velocity and concentration and enhances the temperature. The skin friction reduces and the Nusselt number enhances at the walls while an opposite effect is noticed with that of heat absorbing source.

- An increase in nanoparticles volume fraction $\phi$ enhances the velocity and reduces the temperature and concentration. The Skin friction and Nusselt number reduces with increasing $\phi$. The Sherwood number enhances at the wall $\mathrm{y}=+1$ and reduces at $\mathrm{y}=-1$

- An increase in Forchhemir number $\Delta$ reduces the velocity, temperature and concentration reduces with increasing $\Delta$. Skin friction and Nusselt number reduces with $\Delta$ at the walls.

\section{REFERENCES}

[1] Al-Nimir, M.A., Haddad, O.H: Fully developed free convection in open-ended vertical channels partially filled with porous material, Journal Porous Media, V2, pp.179-189(1999).

[2] Barletta,A,Celli,M and Magtyari,E and Zanchini,E:Buoyany MHD flows in a vertical channel the levitation regime Heat and Mass Transfer, V.44,pp.1005-1013(2007).

[3] Barletta,A,Magyari,E and Kellaer,B:Dual mixed convection flows in a vertical channel .,Int.J.Heat and Mass Transfer,V.48,pp.4835-4845(2005).

[4] Barletta,A:Analysis of combined forced and free flow in a vertical channel with viscous dissipation and isothermal-isoflux boundary conditions, ASME.J.Heat Transfer ,V,121,pp.349-356(1999).

[5] Buongiorno, J: Convective transport in nanofluids, ASME J. Heat Transfer 128 (2006) 240-250.

[6] Cebeci,T,Khattab,A.A and LaMont,R: Combined natural and forced convection in a vertical ducts,in:Proc. $7^{\text {th }}$ Int. Heat Transfer Conf.,V.3,pp.419-424(1982).

[7] Choi, S.U.S., Zhang, Z.G., Yu, W., Lockwood, F.E., and Grulke, E.A: Anomalously thermal conductivity enhancement in nanotube suspensions, Appl. Phys. Lett. 79 (2001) 2252-2254.

[8] Das, S., Jana, R.N., and Makinde, O.D: Transient natural convection in a vertical channel filled with nanofluids in the presence of thermal radiation.

[9] Das, S.K., Choi, S.U.S., Yu, W., Pradeep, T: Nanofluids: Science and Technology, Wiley, New Jersey, 2007.

[10] Datta,N anfd Jana,R.N: Effect of wall conductance on hydromagnetic convection of a radiation gas in a vertical channel., Int.J.Heat Mass Transfer,V.19,pp.1015-1019(1974).

[11] Daungthongsuk, W., Wongwises, S: A critical review of convective heat transfer nanofluids, Renew. Sustain. Energy Rev. 11 (2007) 797-817.

[12] Fakour, M., Vahabzadeh, A., Ganji, D.D: Scrutiny of mixed convection flow of a nanofluid in a vertical channel, Case Stud. Therm. Eng. 4 (2014) 15-23.

[13] Gill,W.N. and Del Casal, A: A theoretical investigation of natural convection effects in a forced horizontal flows, AICHE J,V.8,pp.513-518(1962).

[14] Greif,R,Habib,I.S and Lin,J.c:Laminar convection of a radiating gas in a vertical channel,J.Fluid.Mech.,V.46,p.513(1971).

[15] Grosan, T., Pop, I: Fully developed mixed convection in a vertical channel filled by a nanofluid, J. Heat Transfer 134 (8) (2012) 082501.

[16] Gupta,P.S and Gupta,A.S:Radiation effect on hydromagnetic convection in a vertical channel.,Int.J.heat Mass Transfer,V.127,pp.1437-1442(1973).

[17] Jang, S.P., Choi, S.U.S: Effects of various parameters on nanofluid thermal conductivity, ASME J. Heat Transfer 129 (2007) 617-623.

[18] Kang Ki, J.Y.T., Choi, C.K.: Analysis of convective instability and heat transfer characteristics of nanofluids, Phys. Fluids 16 (2004) 2395-2401. 
[19] Kiblinski, P., Phillpot, S.R., Choi, S.U.S., Eastman, J.A: Mechanism of heat flow is suspensions of nanosized particles (nanofluids), Int. J. Heat Mass Transfer 42 (2002) 855-863.

[20] Maghrebi, M.J., Nazari, M., Armaghansi, T: Forced convection heat transfer of nanofluids in a porous channel, Transp. Porous Media 93 (2012) 401-413.

[21] Maïga, S.E.B., Nguyen, C.T., Galanis, N., Roy, G: Heat transfer behaviours of nanofluids in a uniformly heated tube, Superlatt. Microstruct. 35 (2004) 543-557.

[22] Maïga, S.E.B., Palm, S.J., Nguyen, C.T., Roy, G., Galanis, N: Heat transfer enhancement by using nanofluids in forced convection flows, Int. J. Heat and Fluid Flow 26 (2005) 530-546.

[23] Nield, D.A., Kuznetsov, A.V: Forced convection in a parallel -plate channel occupied by a nanofluid or a porous medium saturated by a nanofluid, Int. J. Heat Mass Transfer 70 (2014) 430-433.

[24] Ostrach, S:Combined natural and forced convection laminar flow and heat transfer of fluid with and without heat sources in channels with linearly varying wall temperature, NACA TN,3141,(1954).

[25] Polidori, G., Fohanno, S., Nguyen, C.T: A note on heat transfer modeling of Newtonian nanofluids in laminar free convection, Int. J. Therm. Sci. 46 (2007) 739-744.

[26] Rossi di Schio, E., Celli, M., Barletta, A: Effect of Brownian diffusion and thermophoresis on the laminar forced convection of a nanofluid in a channel, ASME J. Heat Transfer 136 (2013) 022401.

[27] Sacheti, N.C., Chandran, P., Singh, A.K., Bhadauria, B.S: Transient free convective flow of a nanofluid in a vertical channel, Int. J. Energy Technol. 4 (2012) 1-7.

[28] Seyyedi, S.M., Bararnia, H., Ganji, D.D., Gorji-Bandpy, M., Soleimani, S: Numerical investigation of the effect of a splitter plate on forced convection in a two dimensional channel with an inclined square cylinder, Int. J. Therm. Sci. 61 (0) (2012) 1-14.

[29] Sheikholeslami, M., Ganji, D.D: Magnetohydrodynamic flow in a permeable channel filled with nanofluid, Sci. Iran. B 21 (1) (2014) 203-212.

[30] Sheikholeslami, M., Gorji-Bandpy, M., Ganji, D.D., Soleimani, S., Seyyedi, S.M: Natural convection of nanofluids in an enclosure between a circular and a sinusoidal cylinder in the presence of magnetic field, Int. Commun. Heat Mass Transfer 39 (9) (2012) 1435-1443.

[31] Sheikholeslami, M., Hatami, M., Ganji, D.D: Analytical investigation of MHD nanofluid flow in a semiporous channel, Powder Technol. 246 (0) (2013) 327-336.

[32] Soleimani, S., Sheikholeslami, M., Ganji, D.D., Gorji- Bandpay, M: Natural convection heat transfer in a nanofluid filled semi-annulus enclosure, Int. Commun. Heat Mass Transfer 39 (4) (2012) 565-574.

[33] Sulochana C and Ramakrishna G N: Effect of Heat Sources on Non-Darcy Convective Heat and Mass Transfer Flow of CuO-Water and Al2O3-Water Nanofluids in Vertical Channel, International Journal of Emerging Trends in Engineering and Development, Issue 7, Vol. 3 (May 2017), ISSN 2249-6149, http://www.rspublication.com/ijeted/ijeted_index.htm

[34] Trisaksri, V., Wongwises, S: Critical review of heat transfer characteristics of nanofluids, Renew. Sustain. Energy Rev. 11 (2007) 512-523.

[35] Wang, X., Xu, X., Choi, S.U.S: Thermal conductivity of nanoparticle fluid mixture, J. Thermophys. Heat Transfer 13 (1999) 474-480.

[36] Xu Hang, Pop, I: Fully developed mixed convection flow in a vertical channel filled with nanofluids, Int. Commun. Heat Mass Transfer 39 (2012) 1086-1092.

[37] Xu, H., Fan, T., Pop, I: Analysis of mixed convection flow of a nanofluid in a vertical channel with the Buongiorno mathematical model, Int. Commun. Heat Mass Transfer 44 (2013) 15-22. 


\section{AUTHORS' BIOGRAPHY}

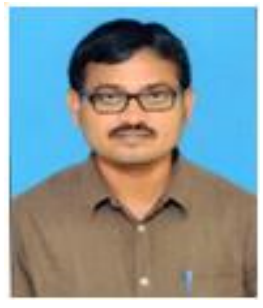

MADDURU SUDHAKARA REDDY, obtained M.Sc. Mathematics in 2006 from S.V. University, Tirupati, Andhra Pradesh, India, and B.Ed in Bangalore University in 2003.I had been working as research scholar in Applied Mathematics S.K.University, Anantapuramu since 2016.My area of specialization is fluid mechanics, heat \& mass transfer, biomechanics, nano technology. I have published three papers in different National and International journals in Applied Mathematics. I have participated in National conferences in India.

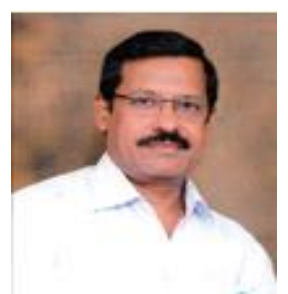

Dr.A.MALLIKARJUNA REDDY, is currently working as a professor in the Department of Mathematics, S.K.University, Anantapuramu, A.P, India. A Doctorate degree holder in mathematics from the same university and has an extensive experience of about 33 years in research and 29 years in teaching for post graduate students. He has published more than 30 research papers in National and International journals and conferences and authored for one Engineering Mathematics. His areas of interest include Fluid Dynamics, Graph Theory and Reliability Theory. He is a member of the Board of Studies in Mathematics for various prominent universities. He held several administrative position in the university and currently he is an Executive Council Member since 2016.

Citation: Sudhakara Reddy, M.\& Prof. A. Mallikarjuna Reddy (2019). Effect of Magnetic Field on Fluid flow and Heat Transfer of a Nanofluid in a Vertical Channel with Heat Sources. International Journal of Scientific and Innovative Mathematical Research (IJSIMR), 7(1), pp.17-28.http://dx.doi.org /10.20431/23473142.0701004

Copyright: () 2019 Authors, This is an open-access article distributed under the terms of the Creative Commons Attribution License, which permits unrestricted use, distribution, and reproduction in any medium, provided the original author and source are credited. 